\title{
Erratum: Hypercube Stacking: A Potts-Spin Model for Surface Growth ${ }^{1}$
}

\author{
Bruce M. Forrest ${ }^{2}$ and Lei-Han Tang ${ }^{3}$
}

The following corrections should be made to the above article.

1. Figure 7 should be replaced by the figure given here. The caption of the figure is as before.

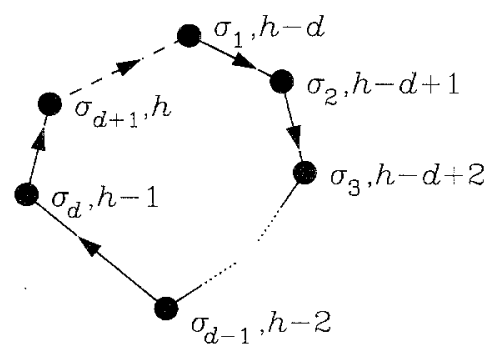

2. The sentence starting from the bottom of p. 195 should read: "Now around any elementary plaquette of the lattice (see Fig. 7) there can be no net height change, implying the existence of one frustrated bond $\Delta h=-d$ (assuming we traverse the plaquette in the direction of the $\hat{\mathbf{e}}_{\alpha}^{\|}$), and $d$ satisfied bonds, $\Delta h=1 . "$

3. On p. 201, footnote 4 is not entirely accurate. Each sublattice updating itself satisfies the detailed balance condition, so that it does not matter how different sublattices are sampled.

We wish to thank H. Leschhorn and R. Németh for bringing the above errors to our attention.

\footnotetext{
${ }^{\mathrm{i}}$ This paper originally appeared in J. Stat. Phys. 60:181 (1990).

${ }^{2}$ Institut für Theoretische Physik, Universität Heidelberg, D-6900 Heidelberg, Germany.

${ }^{3}$ Fakultät für Physik und Astronomie, Ruhr-Universität Bochum, D-4630 Bochum, Germany.
} 